\title{
Pulmonary hypertension associated with non-cirrhotic portal hypertension in systemic lupus erythematosus
}

\author{
Desmond Woolf, Michael D. Voigt ${ }^{1}$, Kasimir Jaskiewicz ${ }^{1}$ and Azga A. Kalla
}

\begin{abstract}
Rheumatic Diseases Unit and ${ }^{1} M R C / U C T$ Liver Research Centre and Department of Medicine, University of Cape Town, Observatory, 7925, South Africa
\end{abstract}

\begin{abstract}
Summary: A case of non-cirrhotic portal hypertension in a patient with systemic lupus erythematosus, the first to our knowledge, is described. Severe pulmonary hypertension was associated with the portal hypertension and with markers of active auto-immunity. Pulmonary hypertension has not previously been associated with non-cirrhotic portal hypertension. The coexistence of vasculopathy of the portal and pulmonary vascular beds in this patient with active autoimmunity supports the postulate that portal-pulmonary hypertension may be immunologically mediated.
\end{abstract}

\section{Introduction}

Although liver disease occurs in up to $20 \%$ of patients with systemic lupus erythematosus (SLE), ${ }^{1}$ non-cirrhotic portal hypertension (NCPH), characterized histologically by portal fibrosis and obliterative lesions of the terminal hepatic veins but well-maintained hepatic architecture, ${ }^{2,3}$ has not, to the best of our knowledge, previously been documented in SLE.

The frequency of primary pulmonary hypertension in patients with portal hypertension is at least $2 \%,{ }^{4}$ which is many times greater than the prevalence in the general population. Pulmonary hypertension has been associated with cirrhosis and portal vein thrombosis, but has not previously been documented in a case of non-cirrhotic portal hypertension. ${ }^{5}$ The cause of the pulmonary hypertension associated with portal hypertension remains obscure but an association with autoimmune phenomena has been suggested. ${ }^{6}$

The case presented documents portal and pulmonary hypertension in a patient with markers of active autoimmunity but absent lupus anticoagulant and supports the postulate that autoimmunity may play a role in inducing the pulmonary hypertension found in patients with portal hypertension.

\section{Case report}

A 19 year old female presented in 1982 with a malar rash, arthritis, Raynaud's phenomenon and leukopenia. Anti-nuclear factor (ANF) to a titre of 1 in

Correspondence: Michael D. Voigt, F.C.P.(S.A.), MRC/ UCT Liver Research Centre, K Floor OMB, Groote Schuur Hospital, Observatory, 7925, South Africa. Accepted: 23 June 1993
2,500, positive anti-DNA antibodies, $27 \%$ circulating immune complexes (CIC) and total haemolytic complement (CH50) of $102 \mathrm{U} / 1$ (normal 160-220 $\mathrm{U} / \mathrm{l}$ ) were demonstrated. Systemic lupus erythematosus was diagnosed ${ }^{7}$ and she was treated with non-steroidal anti-inflammatory drugs (Voltaren, Ciba Geigy). Between 1982 and 1985 she received several courses of hydroxychloroquine and oral steroids for flares in disease activity. In 1985 she was again admitted with a lupus flare and renal impairment associated with an active urinary sediment was found. Markers of activity included circulating immune complexes of $41.4 \%, \mathrm{CH} 50$ of $132 \mathrm{U} / \mathrm{l}$, ANF to a titre of 2,500, and C-reactive protein of $5.6 \mathrm{mg} / \mathrm{dl}$ (normal undetectable). Renal biopsy showed diffuse proliferative glomerulonephritis. She was pulsed with intravenous methylprednisone and discharged on prednisone $60 \mathrm{mg} /$ day. Her further course was complicated by numerous admissions to hospital with active disease, associated with low $\mathrm{CH} 50$ and elevated CIC. Gastrointestinal involvement, presenting with diarrhoea resistant to steroids, occurred in 1986, which responded to therapy with cyclophosphamide. Systemic hypertension developed in late 1986 which was controlled with atenolol and frusemide.

Dyspnoea, with a forceful right ventricular lift, loud pulmonary, component of the second heart sound, tricuspid incompetence and right heart failure led to a diagnosis of pulmonary hypertension in February 1989. Mean pulmonary artery pressure was $77 \mathrm{mmHg}$ on echo cardiography. Multiple pulmonary emboli, occult valvular heart and primary lung disease were excluded by ventilation-perfusion radionucleide scanning, echo cardiography and pulmonary function tests. Anti- 
cardiolipin antibodies and Venereal Diseases Research Laboratories (VDRL) tests for syphilis were negative.

Splenomegaly was noted in May 1989 and hepatomegaly, in the absence of liver congestion, was noted 6 months later. Hepatosplenomegaly progressed, with the development of ascites and lower oesophageal varices in 1991. Serum albumin was $35 \mathrm{~g} / \mathrm{l}$, globulin $55 \mathrm{~g} / \mathrm{l}$ prothrombin time (international normalized ratio; INR) 1.1 (normal $1-1.5$ ), alkaline phosphatase 371 (normal 30$115 \mathrm{U} / \mathrm{l})$, gamma glutamyl transpeptidase 404 (normal $0-50 \mathrm{U} / \mathrm{l}$ ), but serum aspartate and alanine transferase activities were normal. Hepatitis B was negative and alpha-1 antitrypsin, copper and iron studies were normal. Liver histology showed typical changes of non-cirrhotic portal hypertension (Figure 1).

\section{Discussion}

Cirrhosis, chronic active hepatitis, granulomatous hepatitis, chronic persistent hepatitis ${ }^{1}$ and focal nodular hyperplasia ${ }^{8}$ have been found in up to $20 \%$ of patients with SLE. We have been unable to find any previous description of non-cirrhotic portal hypertension in SLE which should be considered in patients who have little hepatocellular dysfunction but evidence of portal hypertension or ascites.

Primary pulmonary hypertension occurs markedly more frequently in patients with portal hypertension. ${ }^{4}$ Although in most reported cases of portal-pulmonary hypertension, the portal abnormality is demonstrated before the pulmonary hypertension, several cases have been documented where recognition of the pulmonary hypertension preceded that of the portal hypertension. ${ }^{4,9,10}$ As both pulmonary and portal hypertension may be clinically silent, abnormalities of both portal and pulmonary vascular beds may be present for con-

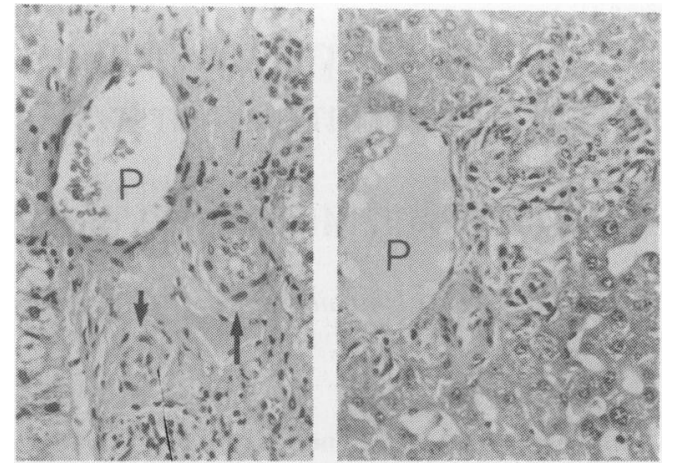

Figure 1 Portal fibrosclerosis of the patient (left) and normal liver (right). Note similar size of portal vein branches (P) and intimal proliferation of the portal arteries (arrows). Haematoxylin and eosin, $\times 180$.

siderable periods before being recognized.

The mechanism of the pulmonary hypertension associated with portal hypertension remains obscure. Primary pulmonary hypertension may occur in systemic lupus erythematosus but is usually associated with the lupus anticoagulant ${ }^{11}$ which was not detected in this case. A total of $5-10 \%$ of patients with portal-pulmonary hypertension have some evidence of autoimmune activity or an associated autoimmune disease. ${ }^{5,6}$ The aetiology of the portal vasculopathy in non-cirrhotic portal hypertension may also be immunologically mediated, as impaired cell-mediated immunity ${ }^{12}$ and disturbed T4/T8 lymphocyte ratios ${ }^{13}$ have been demonstrated in these patients. In this patient, the active rash, complement consumption and high levels of circulating immune complexes indicated an active autoimmune process. This case supports the postulate that the pulmonary hypertension associated with portal hypertension may be immunologically mediated.

\section{References}

1. Runyon, B.A., LaBreque, D.R. \& Anuras, S. The spectrum of liver disease in systemic lupus erythematosis. Report of 33 histologically-proved cases and review of the literature. $\mathrm{Am} \mathrm{J}$ Med 1980, 69: 187-194.

2. Sama, S.K., Bhargava, S., Gopinath, N. et al. Noncirrhotic portal fibrosis. Am J Med 1970, 51: 160-169.

3. Fukuda, K., Kage, M., Arakawa, M. \& Nakashima, J. Portal vein or hepatic vein? A curious aberrant vasculature in the liver with idiopathic portal hypertension. Acta Pathol Jpn 1985, 35: 885-897.

4. Hadengue, A., Benhayoun, M.K., Lebrec, D. \& Benhamou, J.P. Pulmonary hypertension complicating portal hypertension: prevalence and relationship to splanchnic hemodynamics. Gastroenterology 1991, 100: 520-528.

5. Voigt, M.D., Bateman, E.D., Louw, S.J. \& Kirsch, R.E. The pulmonary circulation in liver disease. In: Bomzon, A. \& Blendis, L. (eds) Cardiovascular Complications of Liver Disease. CRC Press, Boca Raton, 1990, pp. 103-124.

6. Morrison, E.B., Gaffney, F.A., Eigenbrodt, E.H., Reynolds, R.C. \& Buja, L.M. Severe pulmonary hypertension associated with macronodular (postnecrotic) cirrhosis and autoimmune phenomena. Am J Med 1980, 69: 513-519.

7. Tan, E.M., Cohen, A.S., Fries, J.F., Masi, J.F. \& Masi, A.T The 1982 revised criteria for the classification of systemic lupus erythematosus. Arthritis Rheum 1982, 25: 1271-1277. 
8. Kiramochi, S., Tashiro, Y., Torikata, C. \& Watanabe, Y. Systemic Lupus Erythematosis associated with multiple nodular hyperplasia of the liver. Acta Pathol Jap 1982, 32: 547-560.

9. Kerbel, N.C. Pulmonary hypertension and portal hypertension. Can Med Ass J 1962, 87: 1022-1026.

10. Squara, P., Dahan, M., Juliard, J.M., Ramond, M.J., Fabiani, B. \& Gourgon, R. Hypertension arterielle pulmonaire revelant une cirrhose hepatique. [Pulmonary arterial hypertension disclosing liver cirrhosis] (letter). Ann Med Interne (Paris) 1987, 138: 377-378.

11. Asherson, R.A., Mackworth-Young, C.G., Boey, M.L. et al. Pulmonary hypertension in systemic lupus erythematosis. $\mathrm{Br}$ Med J 1983, 287: 1024-1025.
12. Malhotra, P., Sharma, B.K., Nayyar, A.K., Sarin, S.K. \& Broor, S.L. Serum immunoglobulins complement and circulating immune complexes in patients with non-cirrhotic portal fibrosis - a comparison with cirrhotics. Proceedings of the 28th Annual Conference of the Indian Society of Gastroenterology, Bhopal, 1987, B2 (abstract).

13. Nayyar, A.K., Sharma, B.K., Sarin, S.K. \& Broor, S.L. Characterization of peripheral blood lymphocytes in patients with non-cirrhotic portal fibrosis - a comparison with cirrhosis and healthy controls. Proceedings of the 28th Annual Conference of the Indian Society of Gastroenterology, Bhopal, 1987, B2 (abstract). 